\title{
Outage probability of coded cooperation for slow fading channel
}

\author{
A. H. M. Almawgani ${ }^{\text {a) }}$ and M. F. M. Salleh ${ }^{\text {b) }}$ \\ School of Electrical and Electronic Engineering, Universiti Sains Malaysia, \\ Seri Ampangan, 14300 Nibong Tebal, Pulau Pinang, Malaysia \\ a) abdulkarem.ld08@student.usm.my \\ b)fadzlisalleh@eng.usm.my
}

Abstract: This letter presents the outage probability of the Reed Solomon (RS) coded cooperation scheme. In this scheme, the codeword of the Users are partitioned into two frames and transmitted through two independent fading channels. The first frame is transmitted directly to the base station. The second frame is transmitted via a partner to the base station. The derived outage expressions confirm that full diversity is achieved by the RS coded cooperation scheme. The analytical outage probability comparisons under various inter-user and uplink channel conditions shed light on the relative virtues of the RS coded cooperation scheme as compared to the RCPC coded cooperation scheme with around 1.5 to $2.5 \mathrm{~dB}$ gains.

Keywords: outage probability, coded cooperation, cooperative diversity, user cooperation, slow fading channels

Classification: Science and engineering for electronics

\section{References}

[1] T. E. Hunter, S. Sanayei, and A. Nosratinia, "Outage analysis of coded cooperation," IEEE Trans. Inf. Theory, vol. 52, no. 2, pp. 375-391, 2006.

[2] Q. Yang and K. Kwak, "Outage probability of cooperative relay in rayleigh fading with unequal-power rayleigh interferers," IEICE Trans. Commun., vol. 91, no. 10, pp. 3360-3363, 2008.

[3] G. D. Menghwar and C. F. Mecklenbrauker, "Outage performance of two users cooperative network coding," Proc. 9th International Symposium on Communications and Information Technology, 2009, ISCIT 2009, pp. 1180-1184, 2009.

[4] A. H. M. Almawgani and M. F. M. Salleh, "Coded cooperation using reed solomon codes in slow fading channel," IEICE Electron. Express, vol. 7, no. 1, pp. 27-32, 2010.

[5] S. Shrestha and K. Chang, "Closed-form solution of outage capacity for cooperative df and af relay network," Wireless Personal Communications, Article ID s11277-009-9761-9, p.15, 2009.

[6] J. Thomas and T. Cover, Elements of information theory, New York: Wiley-Interscience, 2006.

[7] S. M. Alamouti, "A simple transmit diversity technique for wireless communications," IEEE J. Sel. Areas Commun., vol. 16, no. 8, pp. 14511458, 1998. 


\section{Introduction}

The design of cooperative communication system that operates in slow fading environment is commonly measured using the outage probability method. In literature, there has been various outage probability for cooperation strategies introduced [1,2]. For instance in [1], the general outage probability for coded cooperative scheme is derived where the rate compatible punctured convolutional (RCPC) is used. Yang in [2] presents the outage probability for cooperative relay system. In [3], the authors derived a closed form expression for outage probability of a network coded cooperative system.

The coded cooperation scheme presented in [4] which utilizes the Reed Solomon codes for channel coding instead of RCPC is reported to outperform the scheme in [1]. In that work, the authors split the encoded data into two frames. The first frame is transmitted directly to the base station while the second frame is transmitted to the base station via a partner. The authors consider four different possible cooperative cases for transmitting of the second frames $N_{2}$, where the details are readily available in [4].

In this letter, we present the derivation and analysis of the outage probability for the RS coded cooperation scheme presented in [4]. The derived outage expressions are for arbitrary signal-to-noise ratio (SNR). The analytical outage probability comparisons under various inter-user and uplink channel conditions shed light on the relative virtues of the RS coded cooperation scheme as compared to the RCPC coded cooperation scheme with around 1.5 to $2.5 \mathrm{~dB}$ gains.

\section{Outage probability of RS coded cooperation}

The outage probability, $P_{\text {out }}$, is one of the standard performance criteria of diversity systems operating over fading channels $[1,5]$. As a reference, we consider a non-cooperative direct transmission between source and destination with quasi-static fading channel. The capacity of that system characterized by the instantaneous SNR $(\gamma)$ can be expressed by the Shannon formula as $C\left(\gamma_{u, B S}\right)=\log _{2}\left(1+\gamma_{u, B S}\right)$. The channel is in outage if the conditional capacity falls below a selected threshold rate $R$, then the channel will be in an outage condition whenever the $\left.\left[C\left(\gamma_{u, B S}\right)<R\right)\right] \mathrm{b} / \mathrm{s} / \mathrm{Hz}$, or equivalently $\left[\gamma_{u, B S}<2^{R}-1\right][6]$. The outage probability is found by integrating the probability density function (PDF) of over the outage event region [1];

$$
\begin{aligned}
P_{\text {out }} & =\operatorname{pr}\left[\gamma_{u, B S}<2^{R}-1\right]=\int_{0}^{2^{R}-1} \operatorname{P}_{r}\left(\gamma_{u, B S}\right) d \gamma_{u, B S} \\
& =\int_{0}^{2^{R}-1} \frac{1}{\bar{\gamma}_{u, B S}} e^{-\frac{\gamma_{u, B S}}{\bar{\gamma}_{u, B S}}} d \gamma_{u, B S}=1-e^{\left(-\frac{2^{R}-1}{\bar{\gamma}_{u, B S}}\right)}
\end{aligned}
$$

The RS coded cooperation scheme presented in [4] assumes that the total parity $P$ is divided into two parts i.e. $P_{1}=(1-\alpha) P$ and $P_{2}=\alpha P$. These two sets of data are not necessarily to have equal size, and the ratio can have the value in the range of $(0 \leq \alpha \leq 1)$. As detailed in [4], the RS coded cooperation scheme sends data in two frames. The first frame is indicated by $N_{1}=K+P_{1}$. The second frame is represented as $N_{2}=K+P_{2}$, where $K$ is 
the length of the user's original message. In RS coded cooperation scheme the users are allocated overall information rate $R$, which corresponds to $N$ total coded symbols per source block. The base station receives two data frames i.e. from the User (first frame) and Partner (second frame). Both frames contain the original message $K$ and part of parity check. The two copies of $K$ are combined by maximal ratio combined (MRC) algorithm [7]. The ratio of data received out of the total $N$ symbols at the base station from Partner is called the cooperation level indicted by parameter in $\beta$ in Eq. 2. Therefore, the ratio of the received data at the base station for the first frame is given as $(1-\beta)$.

$$
\beta=\frac{N_{2}}{N_{1}+N_{2}}=\frac{K+(1-\alpha) P}{2 K+(1-\alpha) P+\alpha P}=\frac{K+(1-\alpha) P}{2 K+P}
$$

\subsection{RS coded cooperation with free error partner}

If the RS coded cooperation scheme is assumed to have free error during decode and forward at the partner, then the base station will receive the transmitted data from both the source and partner. These two channels are independent $\left(\gamma_{1, B S}\right.$ and $\left.\gamma_{2, B S}\right)$. Thus, the outage events for Users 1 for coded cooperation scheme follow the technique used in [1] where the approximation uses channel capacity as the upper limit.

$$
\begin{aligned}
C_{1, B S}\left(\gamma_{1, B S}, \gamma_{2, B S}\right) & <R, \text { where } \\
C_{1, B S}\left(\gamma_{1, B S}, \gamma_{2, B S}\right) & =(1-\beta) \log _{2}\left(1+\gamma_{1, B S}\right)+\beta \log _{2}\left(1+\gamma_{2, B S}\right) \\
& =\log _{2}\left(1+\gamma_{1, B S}\right)^{(1-\beta)}+\log _{2}\left(1+\gamma_{2, B S}\right)^{\beta}
\end{aligned}
$$

The outage probability, $P_{\text {out }}$, of the outage event in Eq. 3 by

$$
\begin{aligned}
P_{\text {out }_{1}, \text { free error }} & =\operatorname{Pr}\left[C_{1, B S}\left(\gamma_{1, B S}, \gamma_{2, B S}\right)<2^{R}\right] \\
& =\operatorname{Pr}\left[\left[\left(1+\gamma_{1, B S}\right)^{(1-\beta)} \cdot\left(1+\gamma_{2, B S}\right)^{\beta}\right]<2^{R}\right]
\end{aligned}
$$

The inequality in Eq. 4 can be written as;

$\left[\left(1+\gamma_{1, B S}\right)^{(1-\beta)} \cdot\left(1+\gamma_{2, B S}\right)^{\beta}\right]<2^{R}$ Where this expression can be used to solve for $\gamma_{1, \mathrm{BS}}$ as

$$
\gamma_{1, B S}<\frac{2^{\frac{R}{1-\beta}}}{\left(1+\gamma_{2, B S}\right)^{\frac{\beta}{(1-\beta)}}}-1=x_{1}
$$

Since $\gamma_{1, B S}>0$, then

$$
\begin{aligned}
& \frac{2^{\frac{R}{1-\beta}}}{\left(1+\gamma_{2, B S}\right)^{\frac{\beta}{(1-\beta)}}}>1 \\
& 2^{\frac{R}{1-\beta}}>\left(1+\gamma_{2, B S}\right)^{\frac{\beta}{(1-\beta)}}
\end{aligned}
$$

$$
\begin{gathered}
\gamma_{2, B S}<2^{\frac{R}{\beta}}-1=y_{1} \\
P_{\text {out }, \text { free error }=}=\int_{0}^{x_{1}} \int_{0}^{y_{1}} \frac{1}{\overline{\bar{\gamma}_{1, B S}}} e^{-\frac{\gamma_{1, B S}}{\bar{\gamma}_{1, B S}}} \frac{1}{\bar{\gamma}_{2, B S}} e^{-\frac{\gamma_{2, B S}}{\bar{\gamma}_{2, B S}}} d \gamma_{1, B S} d \gamma_{2, B S} \\
=\frac{1}{\bar{\gamma}_{2, B S}} \int_{0}^{y_{1}}\left(1-e^{-\frac{x_{1}}{\bar{\gamma}_{1, B S}}}\right) e^{-\frac{\gamma_{2, B S}}{\bar{\gamma}_{2, B S}}} d \gamma_{2, B S}
\end{gathered}
$$




\subsection{RS coded cooperation with Error partner and reciprocal inter-user channel}

In the case of reciprocal inter-user channels $\left(\gamma_{1,2}=\gamma_{2,1}\right)$ only case 1 and case 2 involve.

- In case 1 , both partners correctly decode each other. This corresponds to the following outage events;

$$
\begin{aligned}
& C_{1,2}\left(\gamma_{1,2}\right)=\log _{2}\left(1+\gamma_{1,2}\right)>R_{2} \\
& C_{2,1}\left(\gamma_{2,1}\right)=\log _{2}\left(1+\gamma_{2,1}\right)>R_{2}
\end{aligned}
$$

where $R_{2}$ is $R / \beta$.

and the outage events for User 1 are given as;

$$
\begin{aligned}
C_{1, B S}\left(\gamma_{1, B S}, \gamma_{2, B S}\right)= & {\left[(1-\beta) \log _{2}\left(1+\gamma_{1, B S}\right)\right.} \\
& \left.+\beta \log _{2}\left(1+\gamma_{2, B S}\right)\right]<R
\end{aligned}
$$

- In case 2 , neither user correctly decodes its partner. Both users transmit additional parity on top of their own data as the second frame. This corresponds to the following outage events;

$$
\begin{aligned}
& C_{1,2}\left(\gamma_{1,2}\right)=\log _{2}\left(1+\gamma_{1,2}\right)<R_{2} \\
& C_{2,1}\left(\gamma_{2,1}\right)=\log _{2}\left(1+\gamma_{2,1}\right)<R_{2}
\end{aligned}
$$

and the outage events for User 1 are given as;

$$
C_{1, B S}\left(\gamma_{1, B S}\right)=\log _{2}\left(1+\gamma_{1, B S}\right)<R
$$

Therefore, the outage probability with error partner for User 1 can be written as;

$$
\begin{aligned}
& P_{\text {out }_{1}, \text { error }}=\operatorname{Pr}\left[\gamma_{1,2}>2^{R_{2}}-1\right] \cdot \operatorname{Pr}\left[\gamma_{2,1}>2^{R_{2}}-1\right] \\
& \cdot \operatorname{Pr}\left[\left(1+\gamma_{1, B S}\right)^{(1-\beta)} \cdot\left(1+\gamma_{2, B S}\right)^{\beta}<2^{R}\right] \\
& +\operatorname{Pr}\left[\gamma_{1,2}<2^{R_{2}}-1\right] \cdot \operatorname{Pr}\left[\gamma_{2,1}<2^{R_{2}}-1\right] \\
& \cdot \operatorname{Pr}\left[\gamma_{1, B S}<2^{R}-1\right] \\
& \text { Pout }_{1, \text { error }}=e^{\left(\frac{1-2^{R_{2}}}{\bar{\gamma}_{1,2}}\right)} e^{\left(\frac{1-2^{R_{2}}}{\bar{\gamma}_{2,1}}\right)} P_{\text {out }_{1}, \text { free error }} \\
& +\left(1-e^{\left(\frac{1-2^{R_{2}}}{\bar{\gamma}_{1,2}}\right)}\right)\left(1-e^{\left(\frac{1-2^{R_{2}}}{\bar{\gamma}_{2,1}}\right)}\right)\left(1-e^{\left(\frac{1-2^{R}}{\bar{\gamma}_{1, B S}}\right)}\right)
\end{aligned}
$$

In the case of reciprocal inter-user channels $\left(\gamma_{1,2}=\gamma_{2,1}\right)$, we can simplify to

$$
P_{\text {out }_{1}, \text { error }}=e^{\left(\frac{1-2_{2} R_{2}}{\bar{\gamma}_{1,2}}\right)} P_{\text {out }_{1}, \text { free error }}+\left(1-e^{\left(\frac{1-2^{2} R_{2}}{\bar{\gamma}_{1,2}}\right)}\right)\left(1-e^{\left(\frac{1-2^{R}}{\bar{\gamma}_{1, B S}}\right)}\right)
$$

\subsection{RS coded cooperation with error partner and independent inter-user Channel}

The general scenario for this condition is when the inter-user channels are independent $\left(\gamma_{1,2}\right.$ and $\left.\gamma_{2,1}\right)$. Case 1 and 2 are explained as in section 2.2 while case 3 and 4 as following; 
- In case 3, User 2 correctly decodes User 1, but User 1 does not correctly decode User 2. This corresponds to the outage events. In the second frame, User 1 and User 2 are both transmitting the same additional parity for User 1, and no additional parity is transmitted for User 2. This corresponds to the following outage events;

$$
\begin{aligned}
& C_{1,2}\left(\gamma_{1,2}\right)=\log _{2}\left(1+\gamma_{1,2}\right)>R_{2} \\
& C_{2,1}\left(\gamma_{2,1}\right)=\log _{2}\left(1+\gamma_{2,1}\right)<R_{2}
\end{aligned}
$$

and the outage events for User 1 are given as;

$$
\begin{aligned}
C_{1, B S}\left(\gamma_{1, B S}, \gamma_{2, B S}\right)= & {\left[(1-\beta) \log _{2}\left(1+\gamma_{1, B S}\right)\right.} \\
& \left.+\beta \log _{2}\left(1+\gamma_{2, B S}+\gamma_{1, B S}\right)\right]<R
\end{aligned}
$$

- Case 4 is identical to Case 3 with the roles of Users 1 and 2 reversed. Thus for the first frame we have the events;

$$
\begin{aligned}
& C_{1,2}\left(\gamma_{1,2}\right)=\log _{2}\left(1+\gamma_{1,2}\right)<R_{2} \\
& C_{2,1}\left(\gamma_{2,1}\right)=\log _{2}\left(1+\gamma_{2,1}\right)>R_{2}
\end{aligned}
$$

and the outage events for User 1 are given as;

$$
C_{1, B S}\left(\gamma_{1, B S}\right)=\log _{2}\left(1+\gamma_{1, B S}\right)<R_{1}
$$

where $R_{1}$ is $R /(1-\beta)$.

When all channels are independent, the overall outage probability for User 1 is given as;

$$
\begin{aligned}
& \text { Put }_{1} \text {,overall } \\
& =P_{\text {out }_{1}, \text { error }}+\operatorname{Pr}\left[\gamma_{1,2}<2^{R_{2}}-1\right] \cdot \operatorname{Pr}\left[\gamma_{2,1}<2^{R_{2}}-1\right] \\
& \quad . \operatorname{Pr}\left[\left(1+\gamma_{1, B S}\right)^{(1-\beta)} \cdot\left(1+\gamma_{1, B S}+\gamma_{2, B S}\right)^{\beta}<2^{R}\right]^{2} \\
& \quad+\operatorname{Pr}\left[\gamma_{1,2}<2^{R_{2}}-1\right] \cdot \operatorname{Pr}\left[\gamma_{2,1}>2^{R_{2}}-1\right] \cdot \operatorname{Pr}\left[\gamma_{1, B S}<2^{R_{1}}-1\right]
\end{aligned}
$$

The inequality in pervious Eq. 16 above can be written as

$$
\gamma_{2, B S}<\frac{2^{\frac{R}{\beta}}}{\left(1+\gamma_{1, B S}\right)^{\frac{(1-\beta)}{\beta}}}-1-\gamma_{1, B S}=x_{2}
$$

Since $\gamma_{1, B S}>0$, then

$$
\begin{aligned}
& \frac{2^{\frac{R}{\beta}}}{\left(1+\gamma_{1, B S}\right)^{\frac{(1-\beta)}{\beta}}}>1+\gamma_{1, B S} \\
& 2^{R}>\left(1+\gamma_{1, B S}\right)
\end{aligned}
$$

$$
\gamma_{1, B S}<2^{R}-1=y_{2}
$$

Due to symmetry, we can obtain an identical expression for User 2 by simply reversing the roles of Users 1 and User 2. Thus the overall outage probability 
of the RS coded cooperation scheme for User 1 is given as;

$$
\begin{aligned}
P_{\text {out }_{1}, \text { vverall }}= & P_{\text {out }_{1}, \text { error }}+e^{\left(\frac{1-2 R_{2}}{\overline{\gamma_{1} 1,2}}\right)}\left(1-e^{\left(\frac{1-2 R_{2}}{\bar{\gamma}_{2,1}}\right)}\right) \\
& \int_{0}^{x_{2}} \int_{0}^{y_{2}} \frac{1}{\bar{\gamma}_{1, B S}} e^{-\frac{\gamma_{1, B S}}{\bar{\gamma}_{1, B S}}} \frac{1}{\bar{\gamma}_{2, B S}} e^{-\frac{\gamma_{2, B S}}{\bar{\gamma}_{2, B S}}} d \gamma_{2, B S} d \gamma_{1, B S} \\
& +\left(1-e^{\left(\frac{1-2 R_{2}}{\bar{\gamma}_{1,2}}\right)}\right) e^{\left(\frac{1-2_{2} R_{2}}{\bar{\gamma}_{2,1}}\right)}\left(1-e^{\left(\frac{1-2 R_{1}}{\bar{\gamma}_{1, B S}}\right)}\right) \\
= & P_{\text {out }_{1}, \text { error }}+e^{\left(\frac{1-2 R_{2}}{\bar{\gamma}_{1,2}}\right)}\left(1-e^{\left(\frac{1-2 R_{2}}{\bar{\gamma}_{2,1}}\right)}\right) \\
& \frac{1}{\bar{\gamma}_{1, B S}} \int_{0}^{y_{2}}\left(1-e^{-\frac{x_{2}}{\bar{\gamma}_{2, B S}}}\right) e^{-\frac{\gamma_{1, B S}}{\bar{\gamma}_{1, B S}}} d \gamma_{1, B S} \\
+ & \left(1-e^{\left(\frac{1-2_{2} R_{2}}{\bar{\gamma}_{1,2}}\right)}\right) e^{\left(\frac{1-2 R_{2}}{\bar{\gamma}_{2,1}}\right)}\left(1-e^{\left(\frac{1-2 R_{1}}{\bar{\gamma}_{1, B S}}\right)}\right)
\end{aligned}
$$

To simplify the expression of $P_{\text {out }_{1}, \text { overall }}$, substitute $P_{\text {out }}$, error in the Eq. 18

$$
\begin{aligned}
P_{\text {out }_{1}, \text { overall }}= & e^{b_{1}} e^{b_{2}} \varphi_{1}+\left(1-e^{b_{1}}\right)\left(1-e^{b_{2}}\right)\left(1-e^{a_{1}}\right)+e^{b_{1}}\left(1-e^{b_{2}}\right) \varphi_{2} \\
& +\left(1-e^{b_{1}}\right) e^{b_{2}}\left(1-e^{a_{2}}\right) \\
& =e^{b_{1}}\left(e^{b_{2}} \varphi_{1}+\left(1-e^{b_{2}}\right) \varphi_{2}\right) \\
& +\left(1-e^{b_{1}}\right)\left[\left(1-e^{b_{2}}\right)\left(1-e^{a_{1}}\right)+e^{b_{2}}\left(1-e^{a_{2}}\right)\right]
\end{aligned}
$$

where

$$
\begin{aligned}
& a_{1}=\frac{1-2^{R}}{\bar{\gamma}_{1, B S}}, a_{2}=\frac{1-2^{R_{1}}}{\bar{\gamma}_{2, B S}}, b_{1}=\frac{1-2^{R_{2}}}{\bar{\gamma}_{1,2}}, b_{2}=\frac{1-2^{R_{2}}}{\bar{\gamma}_{2,1}}, \\
& \varphi_{1}=\frac{1}{\bar{\gamma}_{2, B S}} \int_{0}^{y_{1}}\left(1-e^{-\frac{x_{1}}{\bar{\gamma}_{1, B S}}}\right) e^{-\frac{\gamma_{2, B S}}{\bar{\gamma}_{2, B S}}} d \gamma_{2, B S}, \\
& \text { and } \varphi_{2}=\frac{1}{\bar{\gamma}_{1, B S}} \int_{0}^{y_{2}}\left(1-e^{-\frac{x_{2}}{\bar{\gamma}_{2, B S}}}\right) e^{-\frac{\gamma_{1, B S}}{\bar{\gamma}_{1, B S}}} d \gamma_{1, B S}
\end{aligned}
$$

\section{Results}

This section presents the outage probability that is taken prior MRC process. The RS coded cooperation scheme uses the rate $R=0.5 \mathrm{~b} / \mathrm{s} / \mathrm{Hz}$. Fig. 1 shows the plot for outage probability versus mean uplink SNR $\left(\gamma_{1, B S}=\gamma_{2, B S}\right)$ for various conditions of the inter-user channel. Fig. 1 clearly shows that coded cooperation achieves diversity order two (compared with diversity order one for the non-cooperative transmission) for various inter-user channels conditions. These results indicate the improvement in throughput which coded cooperation provides over the non-cooperative system.

Even when the inter-user channel quality is poor relative to the uplink channels, the RS coded cooperation scheme still provides significant performance improvement. When all channels are in the same conditions, the performance of RS coded cooperation scheme is near to the free error inter-user channel (triangle legend) as shown in Fig. 1.

Fig. 2 compares the outage probability between the RS coded cooperation scheme with RCPC coded cooperation introduced in [1]. For both schemes, the cooperation level $\beta$ is set to 0.5 . The outage probability with the reciprocal inter-user channels which represent by dash line as shown in Fig. 2 is always less than that with independent inter-user channels. The difference in outage probability between the reciprocal and independent inter-user channels is quite small as compared to the overall gain relative to the noncooperative system. The outage probability curves in Fig. 2 shows the RS 


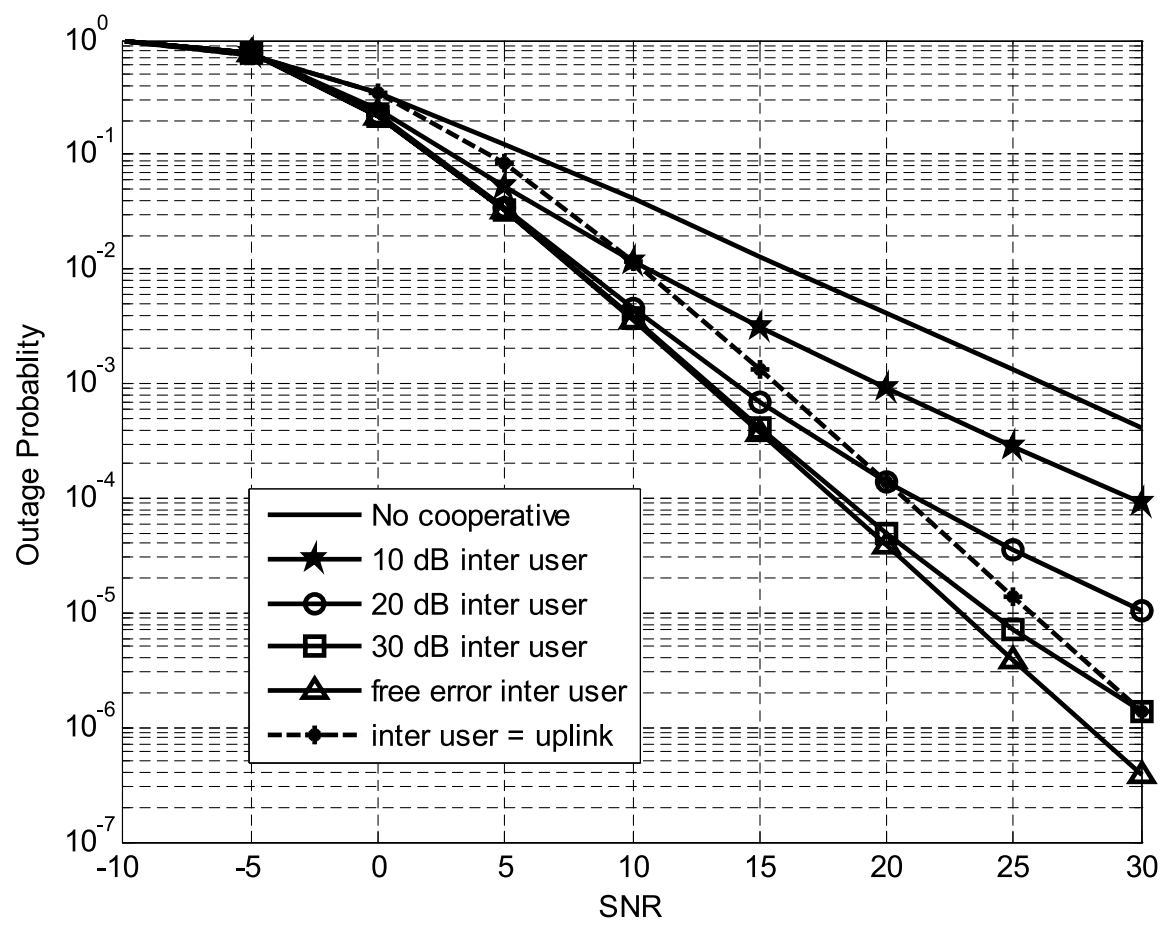

Fig. 1. Outage probability versus $S N R$ for rate $R=0.5$ $\mathrm{b} / \mathrm{s} / \mathrm{Hz}$. Various sets of curves correspond to the inter-user channel mean SNR equal to the mean uplink SNR, fix value 10, 20, $30 \mathrm{~dB}$ and free error inter-user channel.

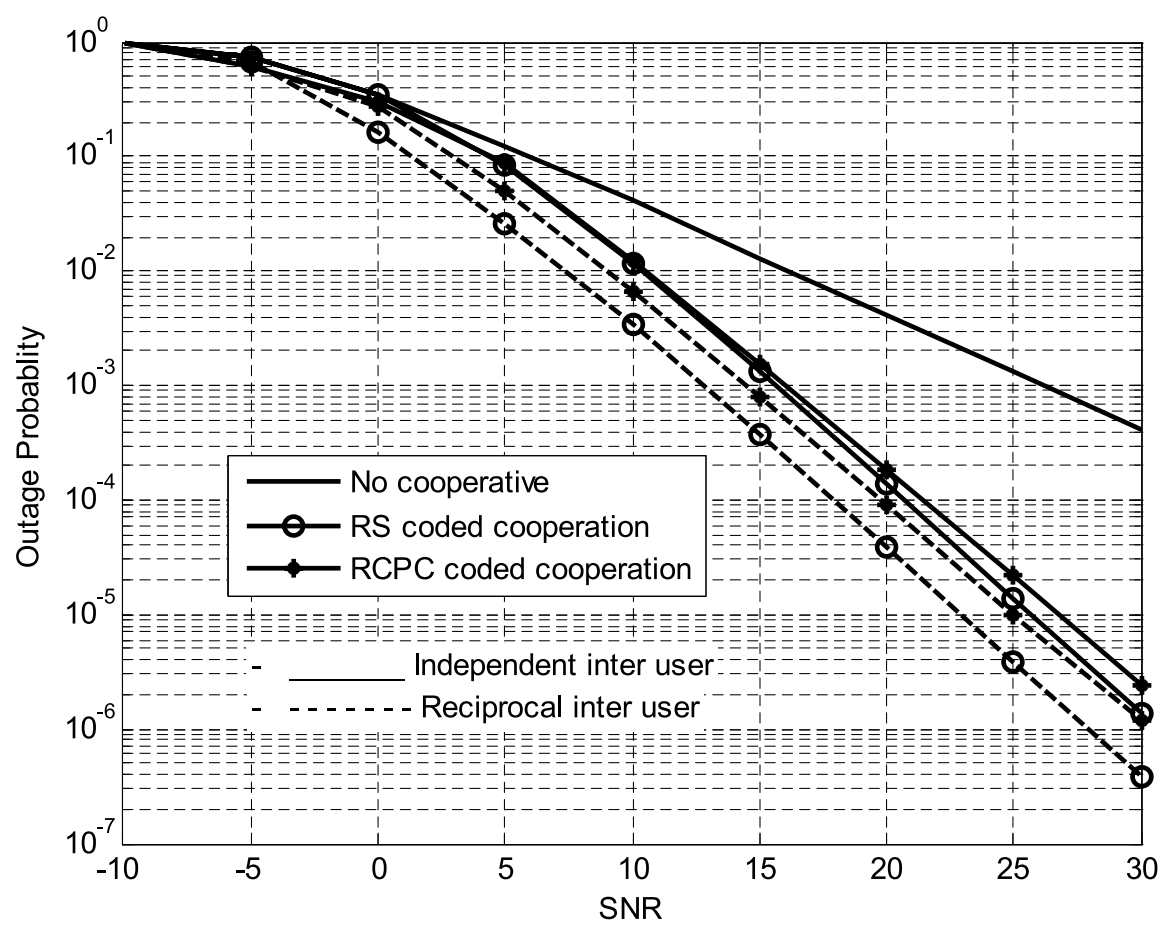

Fig. 2. Outage probability versus $\mathrm{SNR}$ for rate $\mathrm{R}=0.5$ b/s/Hz. All channels have equal mean SNR. Comparison of RS coded cooperation and RCPC coded cooperation. 
coded cooperation outperforms RCPC coded cooperation scheme. Fig. 3 compares the outage probability between numerical results from the mathematics derivation and simulation results for the RS coded cooperation scheme presented in [4]. In the outage probability experiment, the number of missing blocks that at receiver before the MRC process is obtained. The numerical results and experiment curves almost are identical as shown in Fig. 3.

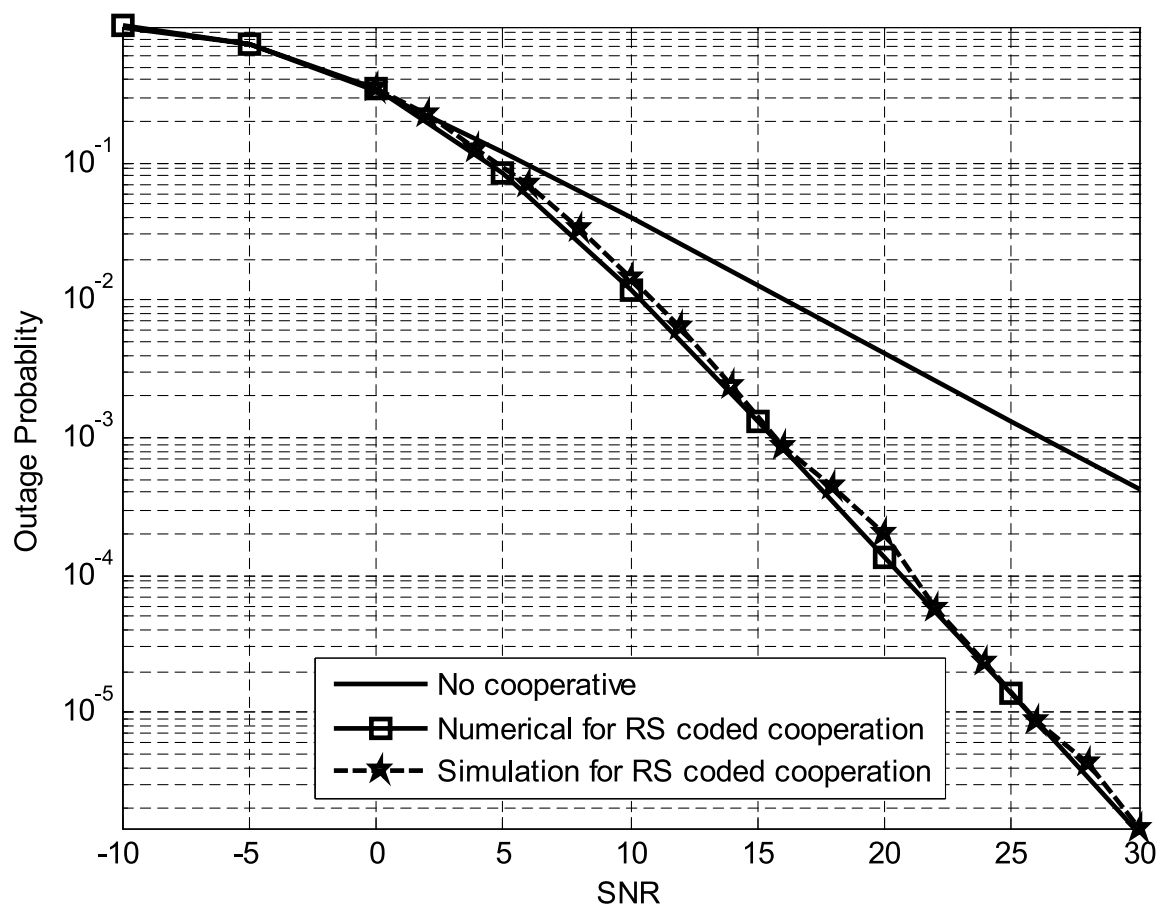

Fig. 3. Outage probability versus $\mathrm{SNR}$ for rate $\mathrm{R}=0.5$ $\mathrm{b} / \mathrm{s} / \mathrm{Hz}$. All channels have equal mean SNR. Comparison of numerical and simulation for RS coded cooperation.

\section{Conclusions}

This letter derives the outage probability of the general coded cooperation scheme in slow Rayleigh fading channels with two cooperating users. The outage probabilities of coded cooperation are derived for four general coded cooperation cases. Simulation and numerical outage probability results demonstrate that the coded cooperation scheme provides significant diversity gains over non-cooperation transmission and related method.

\section{Acknowledgments}

This work is funded in part by MOHE FRGS grant with Grant No. 6071138, USM-RU-PRGS grant with Grant No. 8042003 and USM Fellowship. 